\title{
Braneworld physics from the analog-gravity perspective: finiteness effects
}

\author{
Carlos Barceló ${ }^{1}$ and Antonio Campos ${ }^{1,2}$ \\ ${ }^{1}$ Institute of Cosmology and Gravitation, University of Portsmouth, Portsmouth PO1 2EG, Britain \\ ${ }^{2}$ Institut für Theoretische Physik, Universität Heidelberg, \\ Philosophenweg 16, D-69120 Heidelberg, Germany
}

\begin{abstract}
We study Randall-Sundrum brane models from the viewpoint of condensed matter/quantum optics. Following the idea of analog gravity we obtain effective metrics in fluid and Bose-Einsteincondensate systems mimicking those in the brane framework. We find that the effect of warp factors in the bulk geometry translates into finiteness of the analog systems. As an illustration of this identification we give a new interpretation of the peculiar behaviour of the critical temperature for the splitting of thick branes in warped spacetimes.
\end{abstract}

PACS numbers: 04.50.+h, 11.10.Kk, 04.80.-y, 05.70.Jk, 05.70.Np, 03.75.Fi

\section{INTRODUCTION}

Reasoning by analogy has been frequently the (hidden) seed behind the generation of new ideas in physics. Here we are interested in the existing analogies between gravitational and condensed-matter/quantum-optics realms (see [1] for a review). They offer a bridge for interchanging conceptualizations of phenomena between strongly developed areas. In this letter we want to stress the potential richness of this interrelation in connection with braneworld gravities.

In the last years one of the gravitational configurations that have attracted more attention have been the Randall-Sundrum (RS) brane model with all its variants [2, 3]. Essentially, the geometry of these models consist on a (4+1)-dimensional asymptotically anti-de Sitter (adS) bulk separated into two symmetrical parts through the time development of a 3-dimensional domain wall. When leaving the domain-wall the spacetime becomes progressively warped allowing the existence of a continuum of normalizable Kaluza-Klein modes. The gravitational zero mode reproduces the standard Newton's law on the brane, while the rest introduce short distance deviations form the square law $[3,4]$.

The metrics associated with thick brane generalizations of the RS one-brane model [3] can be written as

$$
d s^{2}=d \eta^{2}+e^{2 A(\eta)}\left[-d t^{2}+d y^{2}+d z^{2}+d w^{2}\right] .
$$

or in conformal coordinates as

$$
d s^{2}=W^{2}(x)\left[d x^{2}-d t^{2}+d y^{2}+d z^{2}+d w^{2}\right],
$$

with $x \equiv \int_{0}^{\eta} e^{-A(\eta)} d \eta$ accounting for the extradimension. For instance, the (thin) RS case corresponds to $A(\eta)=-|\eta| / l$ or $W(x)=1 /(1+|x| / l)$, where $l$ stands for the length scale of the warping which in $n$ dimensions is related with the bulk cosmological constant by $\Lambda=-(n-2)(n-1) / 2 l^{2}$. In this notation a thick domain wall in an asymptotically adS space will correspond to different smoothings of the previous function with the same asymptotic decay $W(x \rightarrow \pm \infty) \sim l /|x|$.
In what follows we will explain how these metrics (more properly their 4-dimensional counterparts which follows from eliminating one of the spatial coordinates on the brane, for instance the $w$ coordinate) can be easily reproduced in an irrotational barotropic fluid and, with a bit more complication, in a Bose-Einstein condensate. On the one side, the existence of a brane in spacetime translates into the existence of a domain wall in the fluid. Many of the concepts appearing in surface critical phenomena, such as partial or complete wetting, will have their own equivalence in braneworld physics. These phenomena will play an important role in understanding the collision and splitting of branes invoked in some braneworld models 5]. On the other side, we will see that the adS nature of the bulk translates into finiteness of the condensed matter/quantum optics system behind the analogy. Analyzing the critical features of the splitting of thick branes in an asymptotically adS bulk one of the authors has found [6] that the critical temperature for complete wetting is lower than that appearing for asymptotically flat spacetimes. From the analog model point of view, this modification of the critical temperature can be seen as a finite-size effect.

\section{FLUID ANALOG}

An irrotational barotropic inviscid fluid can be described by the equation of continuity

$$
\partial_{t} \rho+\nabla \cdot(\rho \mathbf{v})=0
$$

and Euler's equation

$$
\rho \frac{d \mathbf{v}}{d t} \equiv \rho\left[\partial_{t} \mathbf{v}+(\mathbf{v} \cdot \boldsymbol{\nabla}) \mathbf{v}\right]=-\nabla p(\rho)-\rho \boldsymbol{\nabla} \Phi .
$$

Here $\mathbf{v}=\boldsymbol{\nabla} \phi$ is the irrotational velocity of the fluid, $p=p(\rho)$ is the pressure, that only depends on the density $\rho$, and $\Phi$ represents the addition of all possible external potential acting on the fluid. It has been proved that acoustic waves on a background fluid flow configuration 
(indicated by a 0 subscript) behave like a scalar field in a curved background with the following 4-dimensional effective metric [7, 8]

$$
g_{\mu \nu} \equiv \frac{\rho_{0}}{c_{0}}\left[\begin{array}{ccc}
-\left(c_{0}^{2}-v_{0}^{2}\right) & \vdots & -v_{0}^{j} \\
\cdots \cdots \cdots \cdots & \cdot & \cdots \cdots \\
-v_{0}^{i} & \vdots & \delta_{i j}
\end{array}\right] \text {. }
$$

Here the velocity of sound in the fluid is defined as $\left.c_{0}^{2} \equiv(d p / d \rho)\right|_{0}$. We can easily see that in order to reproduce a metric of type (2) we have to setup the velocity of the flow equal to zero $\left(v_{0}^{i}=0\right)$, and to assume a constant velocity of sound; (absorbing this constant into a coordinate redefinition we can set $c_{0}=1$ ). The constancy of the velocity of sound implies that we have to deal with fluids with an equation of state $p=c_{0}^{2} \rho$.

With these requirements the effective metric is obviously conformal to the Minkowski metric $g_{\mu \nu}=\rho_{0} \eta_{\mu \nu}$. To reproduce the conformal factor of a particular brane model one have only to setup the fluid with a density profile of the form

$$
\rho_{0}(x)=W^{2}(x),
$$

and this can be achieved by taking an external potential

$$
\Phi=-2 \ln W(x) .
$$

For instance, the original RS geometry corresponds to

$$
\rho_{0}(x)=\frac{1}{(1+|x| / l)^{2}} ; \quad \Phi=2 \ln (1+|x| / l) .
$$

Given a spacetime geometry it is generically impossible to reproduce it within a fluid analog, even taking advance of the freedom of coordinate choices (a generic 4-dimensional geometry has 6 degrees of freedom and a fluid analog has only two and not completely independent). What we have just seen is that the particularly simple way in which we can write the geometries of Randall-Sundrum type allows us to build analog models for them.

Sonic waves in a background fluid with the above density profile propagate in the same way as a (scalar) field would do in the RS spacetime. The computation of geodesics on this geometry [9] shows that every freefalling test particle is repelled away from the brane unless its trajectory is set initially on the brane itself. A repelled test particle reaches the adS horizon in finite proper time. In the acoustic analog, this proper time corresponds to the relative perception of time in the sonic world. A laboratory observer has a different perception of time based on its underlying Minkowski spacetime. For him the proper time corresponds to the coordinate time $t$ and the test particle would reach the horizon when $t \rightarrow \infty$. Although from the sonic world point of view the geometry might be extended beyond the horizon the reality of the system in the laboratory tell us that this extension is not actually in place. We are dealing with a genuinely incomplete effective geometry.

The behaviour of acoustic waves in these effective geometries captures essential features of the propagation of gravitons in spacetime. Basically, this is so because the background and the acoustic waves are made from the same substance. Therefore, the propagation of acoustic waves in these background geometries can be understood from the braneworld point of view as a combination of zero and massive Kaluza-Klein modes [3]. The zero mode will correspond to wave packets localized on the brane and propagating along the brane. In realistic situations, in which the brane will have some thickness, one expects that one can localize wave packets to move along the brane if only one sets them up initially to have small momenta in the transverse direction. If we associate a wavelength to the transverse momentum $\lambda_{t}$, then this wavelength will have to be much larger than the brane thickness, $\lambda_{t} \gg T$ for localization to occurs. It would be interesting to analyze (and possibly observe) this localization behaviour within the analog models constructed.

Before finishing this section let us just anticipate that Eq. 6, apart from being the key to construct the analog model, will allow an stimulating identification between the effects of warp factors in the bulk geometry and finiteness (finite-size) effects on condensed matter systems.

\section{BOSE-EINSTEIN ANALOG}

Let us analyze now how would it be the construction requirements for braneworld analog models within BoseEinstein condensates. These systems are very promising for building analog models of black holes [11].

An appropriate starting point for describing BoseEinstein condensates in dilute systems is the GrossPitaevskii equation [10]

$$
\mathrm{i} \hbar \frac{\partial}{\partial t} \psi(t, \mathbf{x})=\left(-\frac{\hbar^{2}}{2 m} \nabla^{2}+V_{\text {ext }}+\lambda|\psi|^{2}\right) \psi(t, \mathbf{x}) .
$$

In this approximation the interaction between the bosons in the condensate is modeled by a unique parameter $\lambda=$ $\left(4 \pi \hbar^{2} / m\right) a$, where $a$ is the s-wave scattering length.

By using the Madelung representation for the wave function $\psi(t, \mathbf{x})=\sqrt{\rho(t, \mathbf{x}) / m} \exp [-i \theta(t, \mathbf{x}) / \hbar]$, and defining $\mathbf{v} \equiv \nabla \theta / m$ we can rewrite the (complex) GrossPitaevskii equation as a continuity equation and an Euler equation as in the fluid case. In doing this we can associate to the condensate an equation of state of the form $p=\left(\lambda / 2 m^{2}\right) \rho^{2}$ and consider the potential $\Phi$ in (4) to consist on two parts, $\Phi=V_{\text {ext }} / m+V_{\mathrm{Q}} / m$, the external potential seen from each boson and the so-called quan- 
tum potential

$$
V_{\mathrm{Q}}(\rho) \equiv-\frac{\hbar^{2}}{2 m}\left(\frac{\nabla^{2} \sqrt{\rho}}{\sqrt{\rho}}\right) .
$$

Now, adopting the hydrodynamic approximation, which amount to neglecting the quantum potential (we will comment later on some implications of this approximation), one can proceed with the same steps that in the fluid case to obtain an effective metric. Indeed, it is the same we have obtained before, but now for the sonic perturbations in the condensate 11, 12]. Again, in order that the metric (5) reproduce braneworld metrics (2) we only need to have a constant sound speed $c_{0}=1$ and an specific density profile $\rho_{0}(x)=W^{2}(x)$. However, the constancy of the sound velocity cannot be achived within a standard Bose-Einstein condensate.

A Bose-Einstein condensate can be seen as a particular type of fluid (in the approximation we are dealing with) and so has a particular equation of state. Therefore, the speed of sound depends in a particular way on the density

$$
c_{0}^{2}=\left.\frac{d p}{d \rho}\right|_{0}=\frac{\lambda}{m} \rho_{0} .
$$

Then, a profile in $x$ for the density requires for consistency a counteracting profile for $\lambda$ or for $m$ if we want to keep $c_{0}=$ constant. This could be difficult to achieve in a laboratory but can be envisaged from a theoretical point of view. In condensed matter physics one is used to encounter effective mass calculations such as those for electrons immersed in a band structure (see, for example, [13]). They have been discussed also for excitons (electron-hole pairs) in semiconductors. By doping inhomogeneously the semiconductor one could give place to a point dependent effective mass. Concerning $\lambda$, or what is equivalent the scattering length $a$, there is already many experiments in which the scattering length is changed by using the properties of a Feshbach resonance [14]. Essentially, in the surroundings of a Feshbach resonance the value of the scattering length can be tuned by the application of an external magnetic field. In current experiments this magnetic field is homogeneous over the entire Bose-Einstein condensate and the scattering length is changed only with time. One can imagine a situation in which this modifications occur in an inhomogeneous way without disrupting the condensate itself. From a more general perspective, one can also envisage a situation in which the bosons are immersed in a inhomogeneously doped medium resulting in point dependent interactions.

In principle, given a brane geometry (that is, a warp factor $W$ ), one can build its corresponding analog model by taking the suitable density profile. We only need that the density profile vary smoothly in distances of the order of the healing length defined as $\xi \equiv \sqrt{2} \hbar / m c$. However, this is not possible in the thin brane limit. In this situation the quantum potential (9) cannot be neglected on the brane and thus the hydrodynamic approximation no longer applies there. The quantum potential will give a finite thickness to any physical realization of a brane. Following the discussion on low-momentum approximation in [12], we can see that for waves with wavelengths greater than the healing length, the effective geometrical description is still in place, but with a different speed of sound. The new speed of sound acquires additional (and non-homogeneous in the $x$ direction) contributions coming from the quantum potential. These contributions are of the order of

$$
c_{0} \frac{\rho}{m} \frac{\xi}{T}
$$

Therefore, strictly speaking with the previous procedure one can only construct analog models for branes with $T>\xi$. The effective geometrical (low-energy) notions for the propagation of long wavelength perturbations will show up in a different way in the confined region if $T \sim$ $\xi$. This is the analog to what is expected to happen in proper spacetime brane models. In order to understand the physics of the confined region itself one might need a full-fledged string/M theory.

Another important issue on building analog configurations with BEC is their classical stability. At least for branes with $T>\xi$, stability is guaranteed as in this approximation one does not have to consider the quantum potential and so one is dealing just with a condensate at rest inside a potential well.

\section{BRANES WITH CONSTANT CURVATURE}

Up to now we have discussed how to reproduce a Minkowski brane with an analog model. However, it is also possible to reproduce other types of brane configurations.

Let us consider now a 4-dimensional adS bulk with metric

$$
d s^{2}=\Omega^{2}\left[d x^{2}-d t^{2}+d y^{2}+d z^{2}\right],
$$

where $\Omega=1 /(1+x / l)$ and $x \in(-l,+\infty)$. Starting from this spacetime we can built different types of $\mathbb{Z}_{2}$ (thin) braneworld geometries. If we take $\sigma$ to be the bare brane tension, the condition

$$
\frac{1}{l}=\frac{1}{4} \sigma \kappa^{2}
$$

where $\kappa^{2}$ is the gravitational coupling constant for the bulk, allows the existence of the Minkowski brane we have been analyzing. The $\mathbb{Z}_{2}$ symmetric spacetime that results from inserting a Minkowski brane into the adS bulk is obtained by simply changing $x$ in the metric (12) by $|x|$. If the previous condition is not satisfied, we can introduce an additional length parameter $L$ as

$$
\pm \frac{1}{L^{2}}=-\frac{1}{l^{2}}+\frac{1}{16} \sigma^{2} \kappa^{4} .
$$


The negative sign case allows the existence of an adS brane embbeded into the adS bulk. The metric is given by 15 .

$$
d s^{2}=d \bar{x}^{2}+F^{2}(\bar{x})\left[d \bar{y}^{2}+e^{-2 \bar{y} / L}\left(-d \bar{t}^{2}+d \bar{z}^{2}\right)\right] .
$$

Here $F(\bar{x})=(l / L) \cosh \left[\left(\bar{x}_{h}-|\bar{x}|\right) / l\right]$ with $\bar{x}_{h}$ an integration constant. In the coordinates (12) an adS brane will be located at

$$
\frac{y}{(l+x)}=\sinh \left(\frac{\bar{x}_{h}}{l}\right) \equiv s .
$$

It is worth noticing that $L$ does not appear in this expression. This is because, from the bulk point of view, adS branes with different scales $L$ correspond only to different re-scalings of the coordinates in the brane.

Let us define a function $\alpha_{s}(y)=(y / s)-l$. Then, the metric associated to an adS brane can also be written as in (12) but substituting the conformal factor $\Omega^{2}$ by

$W^{2}(x, y) \equiv \Theta\left(x-\alpha_{s}(y)\right) \Omega^{2}(x)+\Theta\left(\alpha_{s}(y)-x\right) \Omega^{2}\left(x_{s}^{*}(x, y)\right)$,

where $x_{s}^{*}(x, y)$ implements the mirror symmetry across the brane and is defined by

$$
x_{s}^{*}(x, y)=\frac{1-s^{2}}{1+s^{2}} x+\frac{2 s^{2}}{1+s^{2}} \alpha_{s}(y) .
$$

To build the corresponding analog model one will need a density profile and external potential characterized respectively by (6) and (7).

The positive sign case leads us to a de Sitter (dS) brane model with metric 15]

$$
d s^{2}=d \bar{x}^{2}+G^{2}(\bar{x})\left[-d \bar{t}^{2}+e^{2 \bar{t} / L}\left(d \bar{y}^{2}+d \bar{z}^{2}\right)\right] .
$$

with $G(\bar{x})=(l / L) \sinh \left[\left(\bar{x}_{h}-|\bar{x}|\right) / l\right]$. In the coordinates (12) the dS braneworld will be located at

$$
\frac{t}{(l+x)}=\cosh \left(\frac{\bar{x}_{h}}{l}\right) \equiv c .
$$

As before, the warp factor corresponding to the dS brane can be written as

$$
W^{2}(x, t) \equiv \Theta\left(x-\alpha_{c}(t)\right) \Omega^{2}(x)+\Theta\left(\alpha_{c}(t)-x\right) \Omega^{2}\left(x_{c}^{*}(x, t)\right) .
$$

However, in this case we cannot simply identify the corresponding density profile to build an analog model of a dS brane. By looking at the previous formula we see that this profile will depend on time through $\alpha_{c}(t)$ and, therefore, the continuity equation for the fluid will not be satisfied for every $x<\alpha_{c}(t)$. In the coordinates we have analyzed it does not seem physically possible to build this analog model. By choosing a different coordinate system and considering more complicated analog models (see [12]) this problem might by alleviated.

\section{SURFACE CRITICAL PHENOMENA AND FINITENESS EFFECTS}

Phase transitions and critical phenomena play an important role in many areas of physics. In the early universe, objects, such as domain walls, strings, and monopoles are naturally produced in phase transitions. In the context of brane cosmology the importance of critical phenomena appears at an even more fundamental level. It has been suggested that some of the problems in standard cosmology can be solved if our universe is the result of the splitting and later collision of branes [5]. Models of brane inflation can be generically constructed from the collision and subsequent annihilation of branes and anti-branes, being the inter-brane separation the degree of freedom associated with the inflaton field [16]. Brane gases has also been speculated to play a crucial role in understanding the initial singularity and the origin of the spacetime dimensions [17].

From condensed matter we know that the existence of surfaces in a bulk modifies and enriches the phenomenology of critical phenomena. An interesting and illustrative situation is that of semi-infinite systems which undergo a first-order bulk phase transition. As the system is brought near the critical temperature of the transition a disordered phase appears between the free surface and the ordered bulk phase [18]. The disordered phase could wet the free surface partially, forming droplets attached to the surface, or completely, forming a layer of certain thickness. This type of critical phenomena presents a surprising effect: the order parameters of the surface change continuously with the temperature while the order parameter associated with the bulk varies abruptally at the critical temperature. Essentially, this is a consequence of the coexistence of different types of vacuum states as the phase transition is reached. Wetting of surfaces is a critical phenomenon that has been observed in many condensed-matter systems [19]. It has been found to appear also at the deconfined phase of SU(3) Yang-Mills theories 20] and supersymmetric QCD [21].

In a realistic realization of the RS brane scenario one would expect the bulk to be filled with a variety of moduli and gauge fields. If the dynamics of some of these fields is driven by a phase transition the behavior of the embbeded brane could be altered in different physically interesting manners. Recently, it has been shown that the phenomenon of complete wetting also occurs for thick Minkowski branes embbeded in asymptotically adS spacetimes [6]. A remarkable aspect in this case is the existence of a new effective critical temperature due to the presence of an effective negative bulk cosmological constant [6]. The phenomenon can be generically described by a phase transition supporting different discrete ordered phases which coexist with a disordered phase at a certain critical temperature $T=T_{c}$. As this temperature is approached an interface interpolating two different or- 
dered bulk phases breaks into two separated interfaces, and a layer of disordered phase appears between them. Contrary to what happens in asymptotically flat spacetimes, the width of this layer does not diverge for the temperature of vacuum coexistence, $T_{c}$, but for a smaller effective critical temperature $T_{*}$. This novel critical effect is directly linked with the global geometry of the spacetime and can be easily understood kinematically. Since geodesics in RS backgrounds pull matter apart from the brane 9], once the splitting of branes is produced their mutual repulsion makes the growth of the wetting layer to be enhanced.

From the analog-gravity perspective, this modification of the critical temperature can be understood as a type of finite-size effect. We have seen that the geometric warp factor translates into a density profile that diminish progressively (vanishing asymptotically) in the spacial direction that represents the extra dimension. The decay of the profile is such that its integral over this dimension is finite. This means that, in what concerns the extra dimension, a finite amount of fluid has been spread out over an infinite space. In practice the system will finish somewhere; however, with the appropriate decay one can stretch the system as much as desire. The finitesize effects we are discussing here could be more properly called finiteness effects. Finite-size effects are ubiquitous in quantum optics and condensed matter. They generically produce modifications of the critical temperatures associated with the thermodynamic (infinite) limit. The modified critical temperatures are observed to scale with the size of the system (in our case, related with the bulk cosmological constant). Here, we want to suggest that many of the finite-size effects studied in these areas, could have an interpretation in the field of brane physics. Also from this viewpoint, it would be interesting to see what can be learned by studying condensed matter/quantum optics models with different types of finite-size effects: different confinement potentials would lead to finiteness or proper finite sizes; periodic conditions would translate into compactification, etc.

\section{SUMMARY}

In this letter we have built analog models for branes in warped spacetimes. They have helped us to established different links between the behavior of braneworlds, and condensed matter/quantum optics systems. We have identified warp factors in geometry with finiteness in analog systems. In our view, the understanding of brane physics (such as the nucleation of bubbles, the splitting and collision of branes, etc.) when they are immersed in a warped spacetime, can get important insights from the analysis of surface critical phenomena in finite-size systems. The analog models we have constructed can guide us in the task of making a faithful translation of these phenomena into the realm of brane physics.

\section{ACKNOWLEDGMENTS}

CB is supported by the EC under contract HPMF-CT2001-01203. AC acknowledges the support of the University of Portsmouth, the Univertität Heidelberg and the Alexander von Humboldt Foundation.

[1] M. Novello, M. Visser, and G. E. Volovik (Eds), "Artificial black holes" (World Scientific, Singapore, 2002).

[2] L. Randall and R. Sundrum, Phys. Rev. Lett. 83, 3370 (1999).

[3] L. Randall and R. Sundrum, Phys. Rev. Lett. 83, 4690 (1999).

[4] J. Garriga and T. Tanaka, Phys. Rev. Lett. 84, 2778 (2000).

[5] P. J. Steinhardt and N. Turok, Phys. Rev. D 65, 126003 (2002).

[6] A. Campos, Phys. Rev. Lett. 88, 141602 (2002).

[7] W.G. Unruh, Phys. Rev. Lett. 46, 1351 (1981); Phys. Rev. D 51, 2827 (1995).

[8] M. Visser, Class. Quantum Grav. 15, 1767 (1998).

[9] W. Muck, K. S. Viswanathan and I. V. Volovich, Phys. Rev. D 62, 105019 (2000).

[10] F. Dalfovo, S. Giorgini, L.P. Pitaeveskii, and S. Stringari, Rev. Mod. Phys. 71, 463 (1999).

[11] L. J. Garay, J. R. Anglin, J. I. Cirac and P. Zoller, Phys. Rev. Lett. 85, 4643 (2000); Phys. Rev. A63, 023611 (2001).

[12] C. Barceló, S. Liberati and M. Visser, Class. Quantum Grav. 18, 1137 (2001).

[13] J. M. Ziman, Principles of the theory of solids, (Cambridge University Press, Cambridge, 1972).

[14] J.L. Roberts, N.R. Claussen, S.L. Cornish, and C.E. Wieman, Phys. Rev. Lett. 85, 728 (2000); 1795 (2000).

[15] O. DeWolfe, D. Z. Freedman, S. S. Gubser and A. Karch, Phys. Rev. D 62, 046008 (2000).

[16] G. R. Dvali and S. H. Tye, Phys. Lett. B 450, 72 (1999).

[17] S. Alexander, R. H. Brandenberger and D. Easson, Phys. Rev. D 62, 103509 (2000).

[18] R. Lipowsky, Phys. Rev. Lett. 49, 1575 (1982).

[19] P. G. de Gennes, Rev. Mod. Phys. 57, 827 (1985).

[20] Z. Frei and A. Patkós, Phys. Lett. B229, 102 (1989); T. Trappenberg and U.-J. Wiese, Nucl. Phys. B372, 703 (1992).

[21] A. Campos, K. Holland, and U.-J. Wiese, Phys. Rev. Lett. 81, 2420 (1998); Phys. Lett. B443, 338 (1998); Nucl. Phys. (Proc. Suppl.) 73, 438 (1999). 